\title{
An Effectual Identification and Prevention OF DDOS Attacks in Web Using Divide-And-Conquer Algorithm
}

\author{
T.R KAVITHA ${ }^{1}$, G. VELMAYIL ${ }^{2}$ \\ ${ }^{1}$ Research Scholar, Department of Computer Science, Quaid-e-Millath College for women, \\ Tamilnadu, India \\ ${ }^{2}$ Assistant Professor, Department of computer science, Quaid-e-Millath College for women, \\ Tamilnadu, India
}

E-mail: 'praka1928@gmail.com, ${ }^{2}$ velmayil@yahoo.com

\begin{abstract}
In this modern computerized world, large number of new technologies has been emerging. With the tremendous growth of internet services, websites are becoming indispensable. Websites are the common source through which they are made accessible to all. The possibility of sharing information through networking has been growing in geometrical progression. In this connection it is to be noted network attacks, In other words, DDoS attacks also are growing in equal proportion. Websites become accessible to large number of users, it may sometimes lead to overload of the server due to the maximum utilization. The result is server performance goes down and the processing time becomes slow. Sharing of information is being carried out by means of server and client. The client requests for the data from the server and the server provides the response for the client-request. Here the client can violate the server performance by sending continuous or anomaly requests. The result is the server performance becomes degraded. This paper discusses how best the degradation of the performance can be identified and prevented using some algorithm proposed in the methodology. In this work the blocking and preventing is done using a different mechanism based on the category of the client.
\end{abstract}

Keywords: Server, Client, Response, Request, Degradation, Blocking, Category.

\section{INTRODUCTION}

Of the several means for communication, most commonly used technology is the networking. The information is shared by the methodology of sending and receiving the request and response respectively. In the case of multiple requests to the same server, the server responds to the client request in a FIFO manner. In this case, the server performance can be degraded due to multiple requests sent to the server by the clients. This is termed as attack [1]. This kind of attack may be avoided by means of the technique termed as DDoS (Distributed Denial of Service). When the number of users gets increased accessing the websites, the performance of the server gets down. Due to much burden on the server, the response time gets delayed. When the process becomes slow, the ratio of the users accessing to the site also gets down The result is the server performance becomes degraded. Apart from this, it may also happen due to the attack of Hackers.

Websites become accessible to large number of users through internet, it may sometimes lead to overload of the server due to the maximum utilization. The result is server performance goes down and the processing time becomes slow. Server just accepts all requests, stores it in queue and sends the response continuously and thereby hackers will perform faster and reducing the performance quality of the server by increasing high network traffic. Due to the overload of the server, network traffic will be increases to corrupt server bandwidth. Hence Intruders can easily attack towards its their destination and they make the site less popular by making its performance very slow 
due to heavy traffic and overhead. This kind of performance degradation is termed as Hackers (or) Intruders [7].

Hackers are the persons who abuse or waste the server bandwidth unnecessarily in order to make the server performance low and thus they make the website not to be used by the web users in web service. Thus, to deny the access of the intruders to the website, introduces a new technology of DDoS in a new manner. The proposed scheme is to implement a special kind of technique to recognize and prevent the attack carried out by the hackers and block them from using the site. This is termed as Denial of Service and thus is carried out among the web users and is commonly referred to as Distributed Denial of Service (DDoS) [3]. A denial-of-service attack (DoS attack) or distributed denial-of-service attack (DDoS attack) is an attempt to make a computer resource unavailable to its legitimate users [2].

\subsection{Problem Definition}

The denial-of-service (DoS) attacks consist of an overwhelming quantity of packets being sent to a victim - these packets arrive in such high quantities that some key resource at the victim (network bandwidth, memory or I/O buffers, CPU time to compute responses) is quickly exhausted [14]. The victim subsequently either crashes or spends so much time handling the attack traffic that it cannot attend to its real work, thereby depriving legitimate clients access to the victim. The distributed denialof-service (DDoS) attacks are more sophisticated attacks where multiple sources of traffic try to overwhelm the victim, often leading to a catastrophic failure of the service provided by the victim [17].

In paper [1], it was discussed the client is blocked based upon the number of accesses made by the client. If the number of access exceeds a certain limit, the server would stop responding to the client and thus the client is totally blocked. For example, if this is to be implemented on a commercial organization, there is a possibility of blocking the genuine customer also. Thus this would lead to genuine customer dissatisfaction. To avoid this kind of problem, this paper provides an efficient methodology to block and prevent the user based on the category consideration. In that case the user gets response according to the categorization.

\section{PROPOSED METHOD}

The next step of the proposed work is to categorize the anomaly clients who send the attack. This is carried out with the help of the database maintained in the first step. Based upon the entry in the database, the client category is detected whether they are registered client or nonregistered client. In the case of non-registered client, they are blocked temporarily until the peak period is over. In case of registered client, the client is provided with response inspite of the peak period. In the proposed methodology, two types of counts are maintained. These are Access Count and Warning Count. The Access Count is the count that can be incremented every time when the client sends the request. The Warning Count is the count that can be incremented once when the unregistered client sends anomalous request.

The non-registered client can be blocked temporarily and the access count is incremented by one along with the warning count during the peak period. After the peak period of the server, the client can be unblocked and they are provided with the response. In this kind of processing, the unregistered client can be blocked permanently when the warning count reaches certain limit. Otherwise, their request is to be processed and the response is provided to the client.

The proposed methodology not only prohibits the access of unauthorized users or the non-registered clients, but also prohibits the access of authorized users those who send multiple requests often. This is the core of the problem. Thus the first step in the proposed methodology is categorizing users as authorized user or unauthorized user. The next step is providing response to the authorized users and blocking the unauthorized users. The unauthorized users are further categorized based on two types of counts time-namely, access counts and warning counts. The access count users can be permitted to have access to the server even though they are considered unauthorized users. Thus by implementing this methodology in an organization would help provide both mechanisms such as preventing the unauthorized users and also preventing the server performance becoming degraded.

Once the hacker is identified and blocked, then it has to deny the services for the intruders from accessing the website. This is done by the proposed 
Dr. E. Fariborzi, H. A. Kazemabad / International Journal of Computer Networks and Communications Security, 1 (6), November 2013

DDoS Divide-and-Conquer Algorithm, to prevent them from accessing the website. This proposed methodology consists of algorithm to maintain the user list and to prevent the attacks. The algorithm named DDOS Divide-and-Conquer Algorithm and its explanation is given below.

\subsection{Block Diagram for Identification of Attacks}

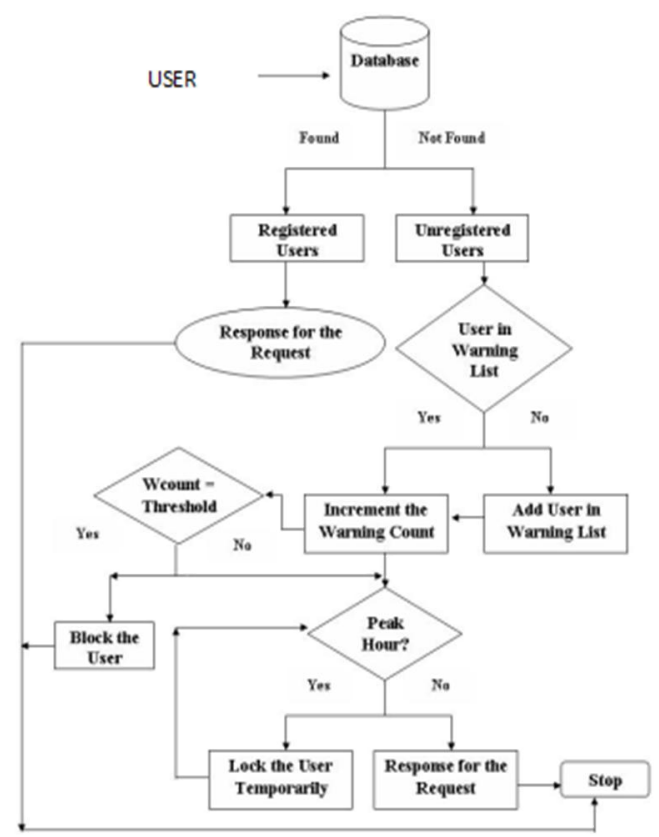

Fig. 1. Identification of attacks under DDoS

\subsection{Flow Chart}

The diagrammatic representation of the flow of the DDoS Divide-and-Conquer algorithm is given as a flowchart below:

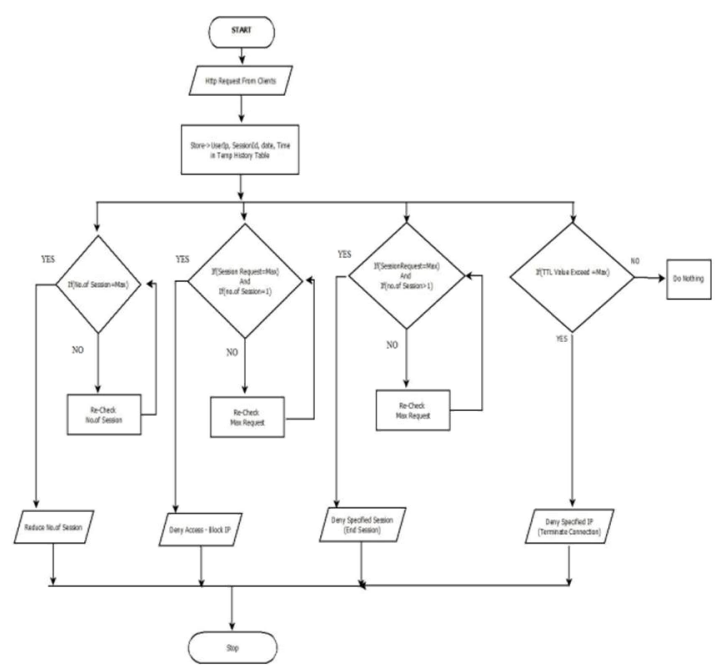

Fig. 2. Prevention of attacks using proposed algorithm

\section{DDOS DIVIDE-AND-CONQUER ALGORITHM}

STEP 1: GET HTTP Request from Clients STEP 2: PARSE [HTTPHEADER]

STEP 3: STORE database entries details in the list IP Addr, SessionID, Date, Time, S.no -> Temp

\section{Track Table}

STEP 4: Use Divide-and-Conquer Search Method (TempTrack, S.no.First, S.no.Last) IP is found STEP 5: If Number of Session value is equivalent to Maximum value

STEP 6: Matched value of step 5 is true then Reduce Number of Session

STEP 7: Matched value of step 5 is false then Recheck Number of Session GOTO ->STEP 8

STEP 8: If (Number of Session $=1$ AND Session Request $=$ High)

STEP 9: Matched value of step 8 is true then Deny Accessibility permissions to IP address

STEP 10: Matched value of step 8 is false Recheck maximum Request GOTO ->STEP 10

STEP 11: If (Number of Session >1 AND Session Request $=$ High)

STEP 12: Matched value of step 11 is true then End specified session

STEP 13: Matched value of step 11 is false then Recheck maximum request GOTO ->STEP 14

STEP 14: If (TTL $>=$ MAX)

STEP 15: Matched value of step 14 is true then the TTL value is exceeded and terminate the connection

STEP 16: Matched value of step 14 is false then do nothing

STEP 17: END

// If the list has 2 or more items

Step 4.1: $\quad$ if (S.no.First $<$ S.no.Last)

// See "Choice of pivot" section below for possible choices

Step 4.2: Choose any pivotIndex such that S.no.First $\leq$ pivotIndex $\leq$ S.no.Last

// Get lists of bigger and smaller items and final position of pivot

Step 4.3: pivotNewIndex:=partition(TempTrack, S.no.First, S.no.Last,pivotIndex)

// Recursively sort elements smaller than the pivot 
Step 4.4: DDOS(TempTrack, S.no.First, pivotNewIndex - 1)

\section{// Recursively sort elements at least as big as the pivot}

Step 4.5: DDOS(TempTrack, pivotNewIndex +1 , S.no.Last)

Step 4.6: function partition(TempTrack, S.no.First, S.no.Last,pivotIndex)

Step 4.7: pivotValue := TempTrack [pivotIndex]

Step 4.8: swap TempTrack [pivotIndex] and TempTrack [S.no.Last]

\section{// Move pivot to end}

Step 4.9: $\quad$ storeIndex $:=$ S.no.First

Step 4.10: for i from S.no.First to S.no.Last - $1 / /$

S.no.First $\leq \mathrm{i}<$ S.no.Last

Step 4.11: if TempTrack[i] < pivotValue

Step 4.12: swap TempTrack[i] and

TempTrack[storeIndex]

Step 4.13: storeIndex $:=$ storeIndex +1

Step 4.14: swap TempTrack[storeIndex] and

TempTrack [S.no.Last]

\section{// Move pivot to its final place}

Step 4.15: return storeIndex

\subsection{Algorithm Explanation}

First step of the algorithm is to get request from the user. In order to detect the intruders, the entry of all users and their activities are maintained as history in the database. The history also contains the information about the users with their corresponding IP address, session id, entry time, date, serial no, and their accessing site. Based on the history, can easily identify all the users accessing the server. Each user entering the internet is assigned a unique IP address. This IP address is also stored in the database along with the users' entry details. The particular user can be identified by this IP address.

This all entry details are stored as history list, if the list is contains $\mathrm{n}$ number of items, then implement a technique called DDoS Divide-andConquer algorithm. The list has to be partitioned by using D\&C Search method and the divided result will be stored in the Temporary track table. By the use of user entry details, check with the number of session value is maximum. If the match returns true value, then reduce number of session. Otherwise, rechecked with maximum number of session. If the details are not matched, then check whether the number of session value is one, which is equivalent to maximum session request value. If the details are matched, then the user is treated as blocked user and the access is denied. Otherwise, rechecked with maximum session request value. If both the matched result returns false then it is compared with, more than one number of session value is equivalent to the maximum session request value, If this is true end that particular or specified session. Otherwise, the user is rechecked with maximum number of session request. Finally, the initial TTL (Time-To-Live) value is compared with the maximum assigned value. If the matched result is true then terminates the connection and end the process. Otherwise, their request is accepted and the response is provided to the user efficiently. Thus this algorithm provides a better method to block and prevent from the intruders from accessing the web page

\section{EXPERIMENTAL RESULTS}

The experimental result of this paper is carried out by implementing the algorithm in a suitable area such as in the commercial website. In this commercial site, we categorize the user into two groups such as: Registered Users and nonRegistered Users. First, the Registered Users are allowed to access the site. They provide the request and wait for the response. To this kind of user, the server provides response without analysing the request. For each and every request of the registered users, the responses are provided. After this, the second category of users namely unregistered users are allowed to access to the server. If this kind of unauthorized user is found accessing to the server during the peak hour, his request is temporarily blocked and this client is added in the list of warning count. These users are again monitored whether they exceed the threshold limit. If they found so, they are categorized under block list permanently. If they are found accessing to the site within the threshold limit, they are allowed to have access to the site. If once the hacker is identified and blocked, then the particular unauthorized user service is not provided to access to deny the services for the intruders from accessing the website. This is done by the proposed DDoS Divide-and-Conquer Algorithm, to prevent them from accessing the website. Thus the experimental setup was constructed and the demonstration was made and the entry is noted to identify and prevent the difference between the attacks made by both kinds of users 


\section{CONCLUSION}

The aim of this paper is to study and devise efficient and practical algorithms to tackle the Websites based distributed denial-of-service attacks, and it focuses to identify and prevent the attack carried out by the hackers and block them from using the site and it also provides how best the degradation of the performance can be prevented by using $\mathrm{N}$ factor DDoS Divide-and-Conquer algorithm proposed in the methodology to improve server performance and deny the accessibility permissions to the hackers.

In this work the blocking is done using a different mechanism based on the user categorization. To improve tracing back the attackers on a global scale is always a difficult and tedious task. For increasing the accuracy of finding attackers, it uses categorizat-ion and Divide-and-Conquer method. To invoke this method by monitoring sever load and network traffic when attains maximal value. Hence there will be no traffic congestion for web users to access the web server with minimal storage overhead and it is effective. Thus the proposed algorithm is suitable for satisfying the organization's requirements. Thus this paper makes an attempt to provide an efficient and well suitable algorithm to identify the attack or threat made by the user on server performance and prevent the server from that kind of attack. In future, this algorithm can be enhanced with proper steps to satisfy large number of requests.

\section{REFERENCES}

[1] Dr. K. Kuppusamy and S. Malathi, "An Effective Prevention of Attacks using GI Time Frequency Algorithm under DDoS", IJNSA journal, Vol. 3, No. 6, November 2011, PP. 249-257.

[2] WONG, Tsz Yeung., On Tracing Attackers of Distributed Denial-of-Service through Distributed Approaches, Ph.D. thesis, The Chinese University of Hong, September, 2007.

[3] M. Muthuprasanna.,"Distributed divide-andconquer techniques for effective DDoS attack defense", G. Manimaran Iowa State University Ames.

[4] K. Park and H. Lee. "On the effectiveness of route-based packet filtering for distributed DoS attack prevention in power-law internets". In Proc. ACM SIGCOMM, San Diego, CA, August 2001.

[5] J. Li, J. Mirkovic, M. Wang, P. Reiher, and L. Zhang , SAVE: source address validity enforcement protocol. In INFOCOM, June 2002.

[6] F. Baker, Requirements for IP version 4 routers. RFC 1812, June 1995.

[7] C. Jin, H. Wang, and K. Shin, Hop-count filtering: An Effective Defense Against Spoofed DDoS traffic. In Proceedings of the 10th ACM conference on Computer and Communications Security, October 2003.

[8] Kihong Park, Heejo Lee, "On the Effectiveness of Probabilistic Packet Marking for IP Traceback under Denial of Service Attack", Network Systems Lab, Department of Computer Sciences, Purdue University, West Lafayette.

[9] Junaid Israr, Mouhcine Guennoun, and Hussein T. Mouftah, "Mitigating IP Spoofing by Validating BGP Routes Updates", IJCSNS, VOL.9 No.5, May 2009, PP 71-76.

[10] Internet Attack Methods and Internet Security Technology, Second Asia International Conference on Modelling \& Simulation, 2008.

[11]Cliff C. Zou, Nick Duffield, Don Towsley, Weibo Gong, "Adaptive Defense Against Various Network Attacks “, University of Massachusetts, AT\&T Labs Research, Florham Park, NJ, 2006.

[12] Guangsen Zhang, Decentralized Information Sharing for Detection and Protection against Network Attacks, Ph.D. thesis, january 2006.

[13] Shigeyuki Matsuda, Tatsuya Baba, Akihiro Hayakawa, and Taichi Nakamura, Design and Implementation of Unauthorized Access Tracing System, Proceedings of the 2002 Symposium on Applications and the Internet (SAINT.02), 2002 IEEE.

[14] William Stallings, Network security Essentials Applications and standards, Fourth Edition, Pearson Education.

[15] Larry Rogers, "What Is a Distributed Denial of Service (DDoS) Attack and What Can I Do About It?" February 2004, http://www.cert.org/homeusers/ddos.html

[16] Chirala Lokesh, B. Raveendra Naick, G. Nagalakshmi, " ETM: a novel Efficient Traceback Method for DDoS Attacks". International Journal of Computer Science and Management Research , Vol 1. Issue 3, October 2012.

[17]Distributed Denial of Service Tools, http://www.cert.org/incident_notes/IN-9907.html

[18]http://en.wikipedia.org/wiki/Divide_and_conqu er_algorithm. 


\section{AUTHOR PROFILES:}

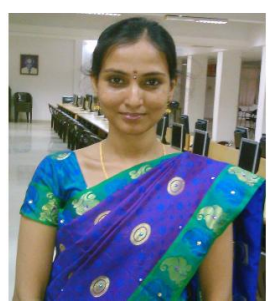

T.R Kavitha received the Master degree in Computer Technology (five years integrated course) from St.Joseph's College of Engineering, Anna Univer-sity, Chennai, Tamilnadu in 2011. She is a research student of Quaid-e-Millath College for Women, Chennai, Tamilnadu. She is pursuing M.Phil degree in Computer Science in the field of computer networks. Currently, she is an Assistant Professor at Meenakshi College for Women, Chennai, Tamilnadu, India. DDoS attacks and Network Security.

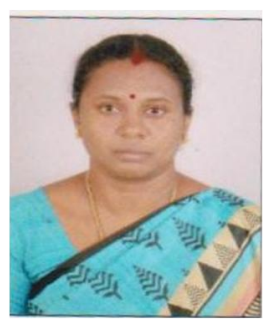

G.Velmayil is an Assistant Professor in Department of Computer Science, Quaid - E -Millath Govt College for Women (Autonomous), Madras University, Chen-nai, Tamilnadu, India. She received the Master degree in Computer applications and M.Phil degree in Computer Science from Bharathidasan University having 17 years of teaching experience. She has organized various workshops, seminars and conferences. She is currently pursuing her Ph.D in the field of computer networks. Her research interest includes DDoS attacks, IP Spoofing and Network Security. 